\title{
Gradhiva
}

\section{Emmanuelle Godeau, $L$ ' Esprit de corps ». Sexe et mort dans la formation des internes en médecine}

Paris, éditions de la Maison des sciences de l'homme, coll. «Ethnologie de la France ", 2007, 303 p.

\section{Julien Bonhomme}

\section{(2) OpenEdition}

\section{Journals}

Édition électronique

URL : http://journals.openedition.org/gradhiva/1772

DOI : $10.4000 /$ gradhiva. 1772

ISSN : 1760-849X

Éditeur

Musée du quai Branly Jacques Chirac

Édition imprimée

Date de publication : 19 mai 2010

Pagination : 221-222

ISBN : 978-2-35744-025-8

ISSN : 0764-8928

Référence électronique

Julien Bonhomme, «Emmanuelle Godeau, L'« Esprit de corps ». Sexe et mort dans la formation des internes en médecine », Gradhiva [En ligne], 11 | 2010, mis en ligne le 09 mai 2010, consulté le 21 septembre 2020. URL : http://journals.openedition.org/gradhiva/1772 ; DOI : https://doi.org/10.4000/ gradhiva.1772

Ce document a été généré automatiquement le 21 septembre 2020.

(C) musée du quai Branly 


\section{Emmanuelle Godeau, L'« Esprit de corps ». Sexe et mort dans la formation des internes en médecine}

Paris, éditions de la Maison des sciences de l'homme, coll. « Ethnologie de la France ", 2007, 303 p.

\section{Julien Bonhomme}

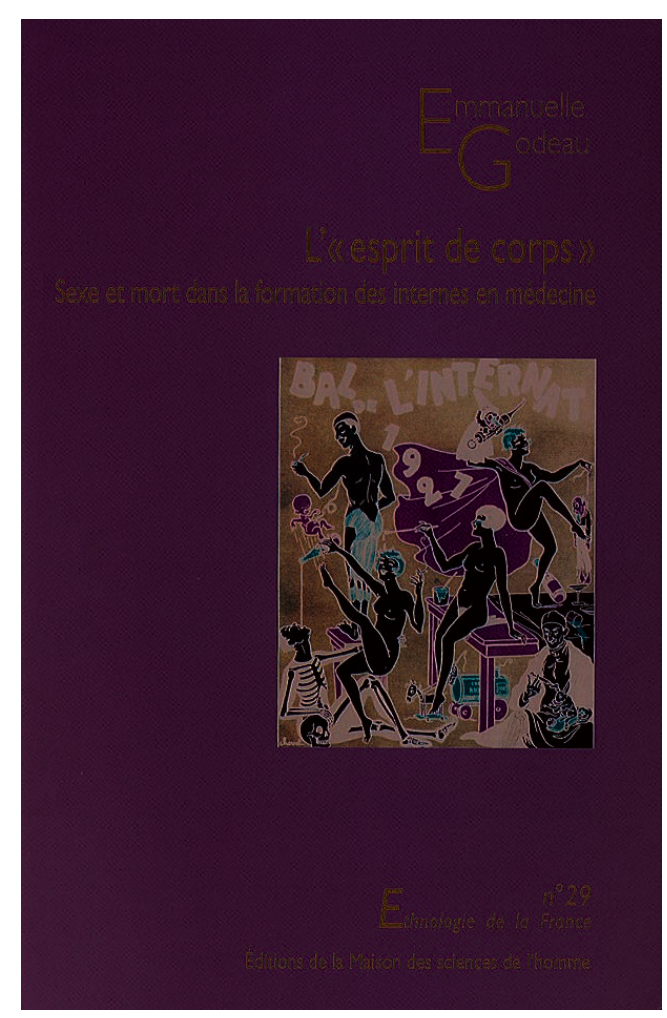

1 Le folklore « carabin » des internes des hôpitaux est au centre de ce bel ouvrage, tiré de la thèse de doctorat d'Emmanuelle Godeau, elle-même médecin et anthropologue. Tout l'intérêt de ce travail est de ne pas envisager les us et coutumes des carabins comme un 
folklore, certes haut en couleur mais somme toute marginal. L'auteur démontre de manière très convaincante qu'il s'agit au contraire d'un élément essentiel - et même constitutif - de la formation des jeunes médecins. En effet, si celle-ci exige l'acquisition de savoirs et de savoir-faire très pointus, elle passe également par une «formation coutumière " qui contribue à façonner l'identité distinctive du corps médical, à lui conférer son « esprit de corps » pour reprendre le titre de l'ouvrage. On ne saurait par conséquent comprendre les modes de socialisation propres à la profession médicale sans faire un détour par une ethnographie des rites carabins.

2 Pour ce faire, l'auteur s'appuie sur un large corpus d'entretiens qu'elle a menés auprès de plusieurs générations d'internes de divers hôpitaux français (ce qui lui permet de prendre en compte les variations géographiques et les évolutions dans le temps), ainsi que sur des observations directes concernant la vie de l'internat. Afin de restituer l'épaisseur culturelle et historique de ce folklore carabin, elle repère des homologies systématiques avec d'autres formes du folklore traditionnel des sociétés européennes, notamment le carnaval et les rites de passage de la jeunesse masculine. De tels rapprochements sont assurément éclairants (même si le lecteur peut parfois se demander s'il faut y voir une simple affinité structurale ou bien une réelle filiation historique) car ils permettent de faire ressortir la cohérence d'ensemble de ce folklore carabin.

3 L'auteur est ainsi amenée à décrire les rites qui scandent la vie de l'internat comme un véritable parcours initiatique grâce auquel les internes acquièrent leurs manières d'être caractéristiques. À travers leur « efficacité symbolique » (c'est-à-dire une façon particulière de transmettre un ensemble de valeurs et de produire une identité sui generis à travers un système spécifique d'actions), ces rites «font" les internes, tout comme une initiation tribale sert à fabriquer les « vrais » hommes. L'efficacité de cette transformation initiatique transparait bien dans les témoignages de ces chefs de service hospitalier qui, plusieurs décennies après leur internat, peuvent déclarer avec fierté : «Interne, c'est mon titre glorieux.» (p. 1) Le plan du livre suit les grandes étapes de ce parcours initiatique.

4 Tout commence par le «baptême ", qui marque l'entrée des impétrants dans l'internat. Avec son bizutage - alliance si particulière de jeu et de sérieux, de transgression et d'obéissance, de secret et d'exhibition -, cette cérémonie a tout du rite initiatique. C'est en effet le baptême qui fait l'interne, plus encore que le concours lui-même : bien des nouvelles recrues déclarent qu'elles ne se sont senties "véritablement » internes qu'à l'issue de leur baptême. Cette production rituelle d'une nouvelle identité inclut même dans certains internats une mise en scène du ré-engendrement des novices: ces derniers passent de la cuisine au réfectoire à travers l'ouverture du passe-plat, qui figure le sexe ouvert d'une gigantesque femme nue peinte sur un mur de la salle de garde. À l'autre bout du parcours initiatique, l'« enterrement» marque la fin de la période de l'internat. Il s'agit d'un rite de séparation mettant en scène la mort de l'impétrant afin qu'il puisse commencer une nouvelle vie (sur le modèle de l'enterrement de vie de garçon) : l'interne est promené dans un cercueil au cours d'une parodie de procession funéraire. La cérémonie constitue une sorte d'apothéose qui célèbre les valeurs de l'internat au moment même où l'interne est amené à le quitter : chacun est en effet censé y manifester à l'excès le type de comportement que l'on peut attendre d'un carabin. 
5 Entre le baptême et l'enterrement s'étend une période «liminaire » centrée autour de la salle de garde, lieu central qui résume à lui seul tout le folklore carabin. Il est d'ailleurs notable que, dans les hôpitaux parisiens, la salle de garde soit également appelée « internat », le terme ayant une remarquable polysémie puisqu'il désigne à la fois un concours, une formation professionnelle, la période de vie qui lui correspond et son espace le plus emblématique. Cette salle de garde est exclusivement réservée aux internes: la frontière spatiale permet en effet de réaffirmer l'identité du groupe à travers l'exclusion des profanes. C'est ce qui explique le refus entêté des internes d'y laisser pénétrer les catégories de personnel hospitalier pourtant les plus proches de la leur : les « externes", les « (internes) provisoires » et même les « internes en médecine générale ", opportunément rebaptisés "résidents " afin de ne justement pas leur conférer le statut privilégié d'interne. Et lorsqu'un externe pénètre exceptionnellement dans la salle de garde à l'invitation d'un interne, il reste en marge du groupe et tous lui font sentir qu'il n'est pas des leurs.

6 La vie de l'internat est rythmée par ses petits rituels quotidiens (les repas en salle de garde) et ses grands événements (les soirées festives appelées «tonus ", ou encore la «revue» de l'internat, spectacle satirique sur le monde hospitalier et ses chefs). L'« économe" est la figure centrale de cet univers: c'est lui qui a en charge l'organisation des repas et, surtout, qui doit faire respecter les traditions, au besoin en infligeant taxes et gages aux contrevenants. L'économe est ainsi tenu d'incarner les manières de salle de garde de façon exemplaire. Celles-ci reposent sur un étonnant mariage de la transgression et de la règle: on doit respecter un code précis pour s'asseoir à table et faire circuler les plats, mais on peut également dévaster le réfectoire au cours d'épiques séances de « projection » de nourriture.

7 La transgression festive se manifeste exemplairement lors des tonus, pendant lesquels les comportements les plus excessifs sont valorisés et encouragés. Cette outrance potache porte notamment sur tout ce qui a trait au corps, au sexe et à la mort: plaisanteries scabreuses, chansons paillardes, fresques obscènes et macabres (des analyses plus précises du corpus des fresques et des chants auraient d'ailleurs été les bienvenues), fêtes alcoolisées ou encore invitation de prostituées (par exemple pour la fellation rituelle du major des internes par une vieille prostituée édentée lors du baptême de l'internat toulousain). C'est bien là ce qui fait la dimension proprement carnavalesque du folklore carabin. Cet univers traditionnellement lié à l'éthos masculin (l'archétype du carabin étant un jeune homme célibataire) se trouve directement affecté par la féminisation progressive de la profession au cours de la seconde moitié du $\mathrm{xx}^{\mathrm{e}}$ siècle. La féminisation ne conduit cependant pas à un effacement pur et simple de la paillardise, mais plutôt à lui donner un nouveau tour, par exemple à travers une surenchère stratégique de certaines femmes internes afin de se faire respecter par leurs collègues masculins.

Évitant le double écueil de la condamnation morale et de la complaisance voyeuriste, l'auteur s'attache à montrer comment cet éthos carabin est directement lié à l'activité professionnelle des internes. Dans l'exercice de leurs fonctions, les médecins ont en effet un accès au corps d'autrui, à sa nudité et à son intimité qui va bien au-delà de ce qui est normalement permis dans le cadre d'une relation entre inconnus. De même, ils sont confrontés à la mort de manière bien plus frontale que la plupart des gens. C'est ce rapport professionnel très particulier au corps et à la mort qui est mis en scène sous une forme exubérante dans le folklore carabin. L'exemple des dissections médicales 
l'illustre bien. Si les travaux pratiques d'anatomie font partie de la propédeutique de tout étudiant en médecine et ne relèvent donc pas de l'internat à proprement parler, l'auteur leur consacre cependant un chapitre entier : la séance de dissection représente en effet le moment inaugural où se noue le rapport à la mort et aux morts constitutif du personnage du médecin. C'est pourquoi son importance est autant symbolique que technique. La leçon de dissection est un véritable rite de passage, vécu par les étudiants comme une «épreuve » qu'ils appréhendent avec autant de crainte que d'excitation et où se joue leur avenir de médecin à travers leur capacité à contrôler leurs émotions face à un cadavre. Tout l'enjeu de la dissection est en effet de réussir à transformer un cadavre inquiétant en une " anatomie », c'est-à-dire en un objet de science médicale.

Cette confrontation avec la mort donne naissance à tout un folklore macabre, typique des carabins : mise en scène avec le cadavre, humour cannibale et nécrophage, etc. Si une approche psychologique en termes de mécanisme de défense contre l'angoisse est sans doute pertinente, l'auteur montre que ce folklore s'inscrit en réalité dans une longue tradition centrée autour de la transgression médicale des interdits liés au corps et à la mort : les premières dissections publiques au $\mathrm{xVI}^{\mathrm{e}}$ siècle ont d'ailleurs été faites sur le cadavre de condamnés à mort, dont le statut «ignoble » permettait justement d'atténuer le caractère transgressif de l'acte. Au XIXe siècle, Gustave Flaubert définit ainsi les « carabins » dans son Dictionnaire des idées reçues : « Dorment près des cadavres. Il y en a qui en mangent. » Et la parenté du médecin avec le boucher, que les carabins d'aujourd'hui mettent en scène dans leurs «batailles de bidoche », est attestée depuis l'Antiquité.

La transgression est donc en réalité double - ce que l'auteur suggère sans l'analyser vraiment. Le médecin est amené à transgresser certains interdits culturels liés au corps et à la mort (largement hérités de la culture chrétienne), mais cette transgression est soigneusement encadrée par une déontologie professionnelle qui la rend acceptable: face à un corps nu ou mort, il doit toujours agir dans le respect de la personne. L'éthos carabin repose sur un redoublement excessif de cette transgression : il met en scène la transgression des limites attachées à la transgression professionnelle. Si l'accès du médecin à la nudité des patients n'est admissible qu'au prix d'une désexualisation du rapport au corps, l'univers carabin repose à l'inverse sur une érotisation outrancière des corps. Le carabin donne ainsi à voir l'image inversée du médecin et de ses normes professionnelles, tout comme le bruit et la saleté de la salle de garde représentent l'envers du silence et de l'hygiène qui doivent régner dans le reste de l'hôpital. « Ainsi, dans la salle de garde une sorte de carnaval permanent se déroule au sein de l'institution hospitalière, à travers une inversion systématique et ritualisée des fondements de cette dernière et des recommandations hippocratiques qui la gouvernent. » (p. 278) La grande qualité de cet ouvrage est en définitive d'avoir su donner au folklore carabin la place qu'il mérite au sein des traditions rituelles des sociétés européennes pour nous faire découvrir un aspect méconnu, et pourtant essentiel, de la profession médicale. 


\section{AUTEUR}

\section{JULIEN BONHOMME}

julienbonhomme@yahoo.fr 